\title{
Topical Piperidine Nitroxide MTS-01
}

National Cancer Institute

\section{Source}

National Cancer Institute. Topical Piperidine Nitroxide MTS-01. NCI Thesaurus. Code C96428.

A topical gel containing a cell permeable hydrophilic piperidine nitroxide with potential radioprotective and antioxidant activity. As a stable, free radical compound, MTS-01 may be able to protect cells against the damaging effects of reactive oxygen species (ROS), upon exposure to ionizing radiation and oxidative stress. The topically applied MTS-01 may protect normal tissue from radiation-induced toxicity, such as radiation dermatitis, during radiation therapy. 